\title{
Meditation Practice and Self-Reported Mindfulness: a Cross-Sectional Investigation of Meditators and Non-Meditators Using the Comprehensive Inventory of Mindfulness Experiences (CHIME)
}

\author{
Claudia Bergomi ${ }^{1}$ • Wolfgang Tschacher $^{1} \cdot$ Zeno Kupper $^{1}$
}

Published online: 9 May 2015

(C) Springer Science+Business Media New York 2015

\begin{abstract}
Mindfulness meditation is generally recognized as the fundamental practice for the development of mindfulness. Accordingly, regular meditation practice is thought to lead to a better capacity to maintain mindfulness during everyday life. Most available studies did not measure the individual amount of experience with meditation practice in detail. In the present study, 683 participants from the general population completed a meditation experience questionnaire and the Comprehensive Inventory of Mindfulness Experiences (CHIME), a scale providing a broad multi-dimensional coverage of mindfulness. Of these, 183 reported currently practicing meditation on a regular basis and provided information about time elapsed since initiation of meditation practice, the amount of current practice, and the techniques used. Results provide evidence for the associations between self-reported mindfulness and meditation practice and suggest that mindfulness is particularly associated with continued practice in the present, rather than with accumulated practice over years. Moreover, no differences in the levels of mindfulness between subgroups practicing with different techniques (Zen, Vipassana, and body movementoriented techniques) could be established, when differences in age and meditation practice were taken into account.
\end{abstract}

Keywords Mindfulness $\cdot$ Meditation $\cdot$ Meditation practice $\cdot$ Meditation technique $\cdot$ Zen $\cdot$ Vipassana $\cdot$ Body movement . Self-report assessment

Claudia Bergomi

bergomi@spk.unibe.ch

1 Department of Psychotherapy, University Hospital of Psychiatry, University of Bern, Bern, Switzerland

\section{Introduction}

Mindfulness is defined as directing attention to the present moment with a particular orientation, which is open to experiences, curious, non-judgmental, non-reactive, decentered (i.e., experiencing one's thoughts and feelings without identifying with them), and insightful (Brown and Ryan 2004; Kabat-Zinn 1994, 2003; Lau et al. 2006; Walach et al. 2006). Mindfulness meditation practice is generally considered the fundamental path for developing mindfulness (Grossman 2010; Kabat-Zinn 1994). The term mindfulness meditation encompasses a wide range of practices which all share the goal of strengthening the practitioner's mindfulness not only during a meditation session but also in daily life (Kabat-Zinn 1994). Mindfulness meditation is widely practiced in the Buddhist tradition and is at the heart of meditation practices such as Vipassana and Zazen. It is however not confined to the Buddhist tradition. Body movement-oriented techniques like walking meditation, Kum Nye, Yoga, or Qigong as well as sitting meditation techniques in which the meditator directs his or her attention to a mantra or visualization are likewise expected to strengthen or facilitate the development of mindfulness (Caldwell et al. 2010; Shelov et al. 2009; Schure et al. 2008).

Previous studies supported the expected relation between the amount of meditation practice and self-report mindfulness. For example, scores on the Mindfulness Attention Awareness Scale (MAAS; Brown and Ryan 2003) significantly differentiated between Zen practitioners and non-meditators and correlated with Zen meditator's perceived engagement in their practice (Brown and Ryan 2003). Mindfulness as measured by the Kentucky Inventory of Mindfulness Skills (KIMS; Baer et al. 2004) was found to be significantly associated with meditation practice in a sample of Vipassana meditators (Falkenström 2010). Soler et al. (2014) reported higher 
mindfulness scores in meditators than non-meditators on all subscales of the Five Facets of Mindfulness Questionnaire (FFMQ; Baer et al. 2006) and the Experiences Questionnaire (EQ; Fresco et al. 2007). They compared different characteristics of meditative practice finding that mindfulness scores were more strongly associated with frequency and months of practice rather than the typical length of a meditation session. In two studies of Baer and colleagues (Baer et al. 2006, 2008), meditation practice in mixed samples of non-meditators (predominantly students) and meditators was significantly correlated with mindfulness as assessed by the total scores of the Freiburg Mindfulness Inventory (FMI; Walach et al. 2006), the KIMS, and the FFMQ. Further studies pointed to significant associations between the FMI, the Southampton Mindfulness Questionnaire (SMQ; Chadwick et al. 2008), and meditation practice in mixed meditators, clinical, and general population samples (Chadwick et al. 2008; Walach et al. 2006). In a study by Carmody and Baer (2008) involving mindfulness-based stress reduction (MBSR) participants, improvements in mindfulness were shown to be associated with the time spent engaging in home meditation practice during the time of the intervention.

Null results however were also reported. For example, meditation practice was not significantly associated with the MAAS in a student sample (Baer et al. 2006). In a study by Falkenström (2010) involving Vipassana meditators, only three of nine mindfulness subscales from the KIMS and the FFMQ were significantly associated with meditation practice.

It is important to note that features inherent to self-report assessment of mindfulness may have influenced results and biased the actual relationships between meditation practice and mindfulness. In fact, the validity of questionnaires in the assessment of everyday mindfulness has been questioned (Chiesa 2013; Grossman 2011). A major concern is the interpretation of mindfulness items. A qualitative study by Belzer et al. (2013) demonstrated that individuals without meditation experience tend to misunderstand 8 of the 14 items of the FMI. Similarly, studies revealed systematic differences in the endorsement of mindfulness items (from the FFMQ, KIMS, and MAAS) that were related to culture, age, or meditation practice, pointing to a possibly idiosyncratic understanding of items by different group of participants (Baer et al. 2007; Christopher et al. 2009; Van Dam et al. 2009). These and related issues may impair the detection of existing associations between meditation practice and mindfulness. On the other hand, meditator expectations regarding the effects of their practice may lead to an overestimation of such associations (Grossman 2008). A further relevant concern relates to the content validity of mindfulness questionnaires. For example, Grossman criticized the MAAS because it assesses mindfulness as the reverse of "self-attribution of inattentiveness during everyday modes of awareness" (Grossman 2011, p. 1035). Rosch (2007) suggested that some mindfulness items may address sanity or reasonableness rather than mindfulness. Bergomi et al. (2013) examined eight validated mindfulness questionnaires and identified nine distinguishable aspects of mindfulness covered in these instruments. Basing on these analyses, the authors constructed a new mindfulness questionnaire, the Comprehensive Inventory of Mindfulness Experiences (CHIME; Bergomi et al. 2014), which provides a broad coverage of the mindfulness construct (see below, Measures).

Current studies frequently fail to provide a detailed description of the relationships between mindfulness and meditation practice. Information about the meditation techniques included in the meditator samples is often missing (e.g., Baer et al. 2004, 2006, 2008; Chadwick et al. 2008), and with a few exceptions (e.g., Lau et al. 2006), no differentiation between beginners and experienced meditators is provided. Moreover, in most studies, meditation practice is operationalized by the number of years since the initiation of practice, whereas current frequency of practice is seldom taken into account.

The present exploratory study offers a more detailed investigation of the associations between mindfulness practice and the development of mindfulness. The measurement of the amount of experience with meditation practice considered different variables such as the techniques used, time elapsed since initiation of the practice, weekly frequency, and average session duration. In order to provide a differentiated account, mindfulness was assessed with the CHIME, a multi-dimensional questionnaire providing a broad coverage of mindfulness. Mindfulness scores were compared between meditators and non-meditators; relationships between mindfulness and amount of meditation practice were analyzed taking into account different variables of meditation practice as well as differences in age and education level. Finally, mindfulness scores were compared between meditators from three mindfulness traditions, who used specific meditation techniques.

\section{Method}

\section{Participants}

Participants in the present study $(N=683)$ were recruited from the general population $(n=443)$ and in MBSR groups $(n=235)$ in Switzerland. In the whole sample of 683 individuals, mean age was 40.1 ( $\mathrm{SD}=13.3), 58.1 \%$ of participants were women. As highest education graduation, most participants reported apprenticeship training or diploma $(33.7 \%)$, followed by advanced technical college (30.7\%), university degree $(20.9 \%)$, university-entrance diploma $(11.9 \%)$, and compulsory school $(2.8 \%)$. Approximately half the participants reported not having any experience with meditation (51.0\%), $18.7 \%$ some experience with meditation in the past and $30.3 \%$ current meditation practice (three participants did not provide enough 
information regarding their meditation practice). Meditation practice was defined broadly including Buddhist meditation techniques, in particular Vipassana and Zen meditation, as well as other techniques such as mantra repetition, Christian spiritual exercises, walking meditation, body scan, relaxation techniques, Yoga, and Qigong.

Two samples were distinguished (Table 1): nonmeditators and meditators. The non-meditator sample $(n=348)$ included participants who reported having no current meditation practice and no experience with meditation in the past. In this sample, mean age was 37.0 $(\mathrm{SD}=12.9)$ and $59.8 \%$ of participants were women. The meditator sample $(n=183)$ comprised all participants with a current regular practice (at least one meditation session per week). Three meditators were excluded because they did not provide information about the time elapsed since start of their practice. Participants in the non-meditator sample were significantly younger than meditators (see Table 1). The two groups did not significantly differ in their distribution of gender and highest education.

In order to build subgroups for the comparison of mindfulness scores across participants training with different meditation techniques, reported techniques were screened. Over 15 different meditation techniques were represented in the meditator sample. Three homogenous subsamples of at least 25 meditators could be identified. Two subsamples included individuals following Buddhist sitting meditation practices (Table 2): Zen $(n=26)$ and Vipassana $(n=38)$. The third subsample included individuals following one (or more) of four body movement-oriented practices: Yoga, Tai Chi, Qigong, and Kum Nye $(n=30)$. The three subsamples significantly differed in their mean age as well as average session duration, one of the provided variables of meditation practice (see Table 2). In both variables, the group mean was highest in the Zen subgroup. The three subgroups did not significantly differ with respect to gender distribution and weekly frequency of practice but showed a marginal significance $(p<.10)$ in meditation experience in months.

\section{Procedure}

Participants of the general population $(n=443)$ were invited by the authors and assistants within their social contexts, in three psychology classes at university, and through a professional association. Participants from MBSR groups $(n=235)$, who were invited by teachers of the Swiss MBSR association, completed procedures during the first week of the intervention. All participants were personally contacted by e-mail or directly by their MBSR teachers. Most participants (91.6 \%) completed procedures online using an internet survey platform. Fifty-six MBSR participants preferred the paper-pencil version of the questionnaire. Data were screened for possible fraudulent data or duplicate responses. There were no discernible duplicates. In a few cases, participants started the questionnaire, interrupted, and started a new form later on. In these cases, data was compared and the complete form was retained.
Table 1 Demographic data, meditation practice, and mindfulness scores in current meditators and non-meditators

\begin{tabular}{lllll}
\hline & Current meditators & Non-meditators & Test of difference & Cohen's $d$ \\
\hline$n$ & 183 & 348 & \\
\% Women & 52.5 & 59.8 & $\chi^{2}=2.62$ & \\
& Mean (SD) & Mean (SD) & $t_{(529)}$ & \\
Age & $43.44(12.73)$ & $37.00(12.87)$ & $5.50^{* * *}$ & \\
Meditation practice & & & & \\
Experience in months & $108.07(117.19)$ & & & \\
Weekly frequency & $4.09(3.32)$ & & & \\
Average session duration & $31.45(23.89)$ & & & \\
CHIME total & $4.79(0.76)$ & $4.31(0.81)$ & $6.66^{* * *}$ & 0.61 \\
Inner awareness & $4.73(0.77)$ & $4.42(1.02)$ & $3.98^{* * *}$ & 0.27 \\
Outer awareness & $4.21(0.86)$ & $3.98(0.86)$ & $3.01^{* *}$ & 0.56 \\
Acting with awareness & $4.01(1.01)$ & $3.45(0.98)$ & $6.17^{* * *}$ & 0.61 \\
Openness & $4.10(0.93)$ & $3.56(0.85)$ & $6.66^{* * *}$ & 0.84 \\
Acceptance & $4.26(0.94)$ & $3.50(0.86)$ & $9.10^{* * *}$ & 0.61 \\
Decentering/nonreact & $4.75(0.86)$ & $4.23(0.85)$ & $6.61^{* * *}$ & 0.50 \\
Insight & $4.32(0.89)$ & $3.91(0.75)$ & $5.35^{* * *}$ & 0.77 \\
Relativity of thoughts & $4.38(0.68)$ & $3.90(0.56)$ & $8.24^{* * *}$ & \\
\hline
\end{tabular}

$S D$ standard deviation; significance levels: $* * p<.01, * * * p<.001$ 
Table 2 Demographic data and meditation practice in the samples varying in their meditation techniques

\begin{tabular}{|c|c|c|c|c|}
\hline & Vipassana & Zen & Body & Test of difference \\
\hline$n$ & 38 & 26 & 30 & \\
\hline \multirow[t]{2}{*}{ \% Women } & 57.9 & 42.3 & 63.3 & $\chi^{2}=2.66$ \\
\hline & Mean (SD) & Mean (SD) & Mean (SD) & $F_{(2,91)}$ \\
\hline Age & $39.32(10.93)$ & $46.88(11.96)$ & $41.23(11.20)$ & $3.57^{*}$ \\
\hline \multicolumn{5}{|l|}{ Meditation practice } \\
\hline Experience in months & $103.95(105.82)$ & $122.31(99.58)$ & $68.67(73.01)$ & $2.37^{\dagger}$ \\
\hline Weekly frequency & $3.82(2.39)$ & $4.84(4.58)$ & $3.33(2.98)$ & 1.51 \\
\hline Average session duration & $32.70(14.17)$ & $49.33(32.86)$ & $32.92(23.37)$ & $4.66^{*}$ \\
\hline Minutes per week & 132.638 (116.99) & $194.52(156.90)$ & $122.75(194.16)$ & 1.73 \\
\hline
\end{tabular}

$S D$ standard deviation; significance levels: ${ }^{\dagger} p<.10,{ }^{*} p<.05$

\section{Measures}

\section{Mindfulness}

Mindfulness was assessed with a newly developed questionnaire that provides a broad multi-dimensional assessment of the construct, the CHIME (Bergomi et al. 2014). The Germanlanguage version was used. The CHIME is based on the conceptualizations of mindfulness underlying eight validated mindfulness questionnaires as well as on theoretical considerations. A preliminary version of the CHIME covered nine aspects of mindfulness that were identified through an overview of eight validated mindfulness questionnaires (Bergomi et al. 2013). The preliminary version was examined psychometrically in a large sample which, together with theoretical considerations, led to the exclusion of items assessing the capacity to verbalize emotions and thoughts (labeling), which is included in the questionnaires KIMS and FFMQ. This decision is supported by recent evidence from two studies involving Zen and Vipassana meditators (cf. Christopher et al. 2014; Falkenström 2010).

The construction of the CHIME put a major focus on items that are as semantically clear as possible in different population subgroups. For example, in line with the results provided by Belzer et al. (2013), in the CHIME construction, ambiguous words such as "experience" and meditation jargon (e. g., FMI 3: "When I notice an absence of mind, I gently return to the experience of the here and now.") were explicitly avoided. Analyses of measurement invariance of the final version of the questionnaire showed no systematic differences in item endorsement that would be related to age, gender, or experience with meditation (Bergomi et al. 2014).

The CHIME provides eight subscales: awareness toward internal experiences (inner awareness), awareness toward external experiences (outer awareness), acting with awareness (acting with awareness), openness to experiences (openness), accepting and non-judgmental orientation (acceptance), decentering and nonreactivity (decentering/nonreact), insightful understanding (insight), and relativity of thoughts (relativity). Examples of items for each subscale are listed in Table 3. The factor structure proved to be stable over three samples, and validity analyses provided good results (Bergomi et al. 2014). The CHIME total score and each subscale showed associations with measures of mental health and well-being in the expected direction. Contrary to studies based on the FFMQ (Baer et al. 2006; Sugiura et al. 2012; Thompson and Waltz 2010), this was the case also in samples mostly inexperienced in meditation. The CHIME seems to provide a comprehensive and robust multi-dimensional assessment of mindfulness and is thus particularly suited for the investigation of differential associations at the level of the individual aspects of mindfulness.

\section{Meditation Practice}

Participants were queried if they had any experience with meditation. If the answer was affirmative, the following questions were presented: 1. "Do you currently meditate?," 2. "Which meditation techniques do you practice?." 3. "How long have you been practicing? (in number of years or months)," 4. "How frequently and how long do you meditate each week? (a) Number of weekly meditation sessions, (b) average duration of a meditation session in minutes." Additionally, participants were given space to write open commentaries in case they felt that the format of the questions did not capture their experience. The following variables of meditation practice were derived from this information: meditation experience in months, weekly frequency of meditation practice, and average session duration. While the first variable represents a retrospective assessment of meditation practice, the two other variables relate to the participant's current meditation practice. The following compound variable of current meditation practice was calculated: minutes of practice per week (weekly frequency $\times$ average session duration). 
Table 3 Example items for the CHIME subscales

\begin{tabular}{ll}
\hline CHIME subscale & Example item \\
\hline Inner awareness & I clearly notice changes in my body, such as quicker or slower breathing. \\
Outer awareness & I notice sounds in my environment, such as birds chirping or cars passing. \\
Acting with awareness & In everyday life, I get distracted by memories, images or reverie (reverse score). \\
Openness & I try to distract myself when I feel unpleasant emotions (reverse score). \\
Acceptance & Even when I make a big mistake, I treat myself with understanding. \\
Decentering/nonreact & When I experience distressing thoughts or images, I am able just to notice them without having to react immediately. \\
Insight & I need to smile when I notice how I sometimes see things as more difficult than they actually are. \\
Relativity of thoughts & It is clear to me that my evaluations of situations and people can easily change. \\
\hline
\end{tabular}

Higher scores for reverse-scored items represent lower levels of mindfulness

\section{Statistical Methods}

The descriptive data of the various groups in the study were compared by $t$ tests (current meditators vs. non-meditators) and $F$ tests (Vipassana, Zen, and body-oriented). Effect sizes were calculated using Cohen's $d$, which provides a measure of standardized mean differences. In the whole meditator sample, the relationships between meditation practice and mindfulness were analyzed in two ways: First, meditation practice as measured by the variables meditation experience in months, weekly frequency, duration of a typical meditation session and the compound variable minutes of practice per week was correlated with the participants' CHIME scores using Pearson's correlations. Second, linear regression analyses were performed including meditation experience in months, weekly frequency, duration of a typical meditation session, age, and education as predictors. In these analyses, the compound variable minutes of practice per week was not included because of its redudancy with the variables weekly frequency and duration of a typical meditation session. Two of the three meditation practice variables, meditation experience in months and weekly frequency, were significantly correlated $(\rho=.35$, $p<.001$ ). Thus, collinearity was screened by diagnostic indices, the tolerance index and the variance inflation factor (VIF). For both indices, values $(T>0.80, \mathrm{VIP}>1.25)$ did not indicate high collinearity.

In order to examine differences between subgroups practicing with different techniques, we used ANCOVA with the three-level categorical variable technique (Vipassana, Zen, body-oriented) as a factor. The variables age, meditation experience in months, and duration of a typical meditation session were included as covariates, as they considerably varied between the subgroups. ANCOVA was only performed in a subsample $(n=94)$ of meditators, which consisted of participants practicing either Zen, Vipassana, or body movementoriented techniques.

According to power analyses, with $\alpha=.05$ and power $(1-\beta)=.80$, sample sizes were sufficient and provided satisfactory sensitivity. In the meditator sample $(n=183)$, an effect size of 0.18 was required in order to detect a significant correlation or a significant regression coefficient. In the ANCOVA $(n=94)$, the effect size needed in order to detect an effect was 0.33 . Statistical analyses were conducted with PASW Statistics 18.0 and power analyses with G*Power 3.1.3.

\section{Results}

Scores of current meditators and non-meditators (Table 1) on the CHIME mindfulness questionnaire were compared. Meditators had significantly higher scores on all variables. The CHIME subscales acceptance, relativity, openness and decentering/nonreact as well as the CHIME total score showed the strongest effect sizes.

Correlations between the amount of meditation practice and CHIME scales were calculated in the whole meditator sample (Table 4). Weekly frequency of meditation practice and meditation experience in months were significantly associated with all CHIME subscales and with the CHIME total score. Typical session duration was significantly associated with all CHIME scales except inner awareness and outer awareness. The associations with the compound variable minutes per week were all significant. All associations were positive, and the strength of associations was weak to moderate. Overall, both the degree of previous as well as current meditation practice was most strongly correlated with the CHIME total score and the subscales inner awareness and decentering/ nonreact. Across all meditation experience variables, the compound variable minutes per week showed the strongest correlations with the mindfulness subscales as well as with the overall score. Of the three non-compound meditation experience variables, weekly frequency showed the strongest associations with mindfulness. Under a strict Bonferroni correction for 36 tests, 14 of the overall 34 significant correlations maintained significance. This applied for all correlation coefficients to the CHIME overall score and decentering/nonreact. 
The relative influence of each meditation practice variable was investigated taking into account differences in age and education level. Thus, linear regression models were calculated for each mindfulness subscale as well as for the CHIME total score (Table 5). In these models, weekly frequency of practice and average session duration were significant predictors for the CHIME total score and for five of the eight CHIME subscales: acceptance, decentering/nonreact, openness, relativity, and insight. Inner awareness was significantly predicted by weekly frequency and outer awareness by meditation experience in months and weekly frequency. Weekly frequency was thus the most consistent predictor of mindfulness scores. The overall regression model of the dependent variable acting with awareness was significant, yet no single predictor in this model reached significance. The models predicting the CHIME total score, inner awareness, and decentering/nonreact showed the largest portions of explained variance.

When group differences in age, average session duration, and meditation experience in months were taken into account, ANCOVA analyses did not detect any significant differences in mindfulness scores across subgroups practicing either Vipassana, Zen, or body movement-oriented techniques (Table 6). In two cases (outer awareness and openness), the technique factor reached marginal significance. In four cases (acting with awareness, acceptance, relativity, and insight), the overall ANCOVA model did not reach significance. Thus overall, results suggest no substantial differences between the meditator subgroups.

\section{Discussion}

The overall goal of the present study was to investigate the relationships between self-reported mindfulness and meditation practice. In particular, the main goal was to provide a differentiated examination covering different aspects of meditation practice and of mindfulness, as well as potential differences arising from the use of specific meditation techniques.

Meditators showed significantly higher scores than nonmeditators on all aspects of mindfulness assessed by the CHIME. The largest differences were found for the CHIME subscales acceptance and relativity, while inner and outer awareness showed significant but small effect sizes. In the meditator sample, mindfulness meditation practice was significantly associated with all aspects of mindfulness assessed by the CHIME. Overall, meditation practice was most strongly correlated with inner awareness and decentering/nonreact. Similarly, the regression models predicting scores in these two variables showed the highest explanation of variance. At the level of the single coefficients, the correlation and regression results displayed different patterns. In the correlational analyses, experience in months and weekly frequency were 
Table 5 Regression analyses showing prediction of mindfulness scores in the whole meditator group

\begin{tabular}{|c|c|c|c|c|c|c|}
\hline & $R^{2}$ & $F$ & $B$ & SE & beta & $t$ \\
\hline Inner awareness & 0.161 & $6.79 * * *$ & & & & \\
\hline Months experience & & & 0.001 & 0.000 & 0.086 & 1.12 \\
\hline Weekly frequency & & & 0.075 & 0.017 & 0.325 & $4.36^{* * *}$ \\
\hline Average session duration & & & 0.004 & 0.002 & 0.133 & $1.93^{\dagger}$ \\
\hline Age & & & 0.001 & 0.004 & 0.015 & 0.20 \\
\hline \multirow[t]{2}{*}{ Education } & & & 0.028 & 0.053 & 0.038 & 0.54 \\
\hline & $R^{2}$ & $F$ & $B$ & SE & beta & $t$ \\
\hline Outer awareness & 0.076 & $2.90^{*}$ & & & & \\
\hline Months experience & & & 0.001 & 0.001 & 0.173 & $2.15^{*}$ \\
\hline Weekly frequency & & & 0.037 & 0.018 & 0.157 & $2.00 *$ \\
\hline Average session duration & & & 0.002 & 0.002 & 0.075 & 1.03 \\
\hline Age & & & -0.002 & 0.005 & -0.030 & -0.39 \\
\hline \multirow[t]{2}{*}{ Education } & & & -0.068 & 0.056 & -0.090 & -1.21 \\
\hline & $R^{2}$ & $F$ & $B$ & SE & beta & $t$ \\
\hline Acting with awareness & 0.103 & $4.09 * *$ & & & & \\
\hline Months experience & & & 0.000 & 0.001 & 0.061 & 0.77 \\
\hline Weekly frequency & & & 0.031 & 0.020 & 0.119 & 1.55 \\
\hline Average session duration & & & 0.005 & 0.003 & 0.138 & $1.93^{\dagger}$ \\
\hline Age & & & 0.010 & 0.005 & 0.147 & $1.96^{\dagger}$ \\
\hline \multirow[t]{2}{*}{ Education } & & & 0.119 & 0.062 & 0.141 & $1.92^{\dagger}$ \\
\hline & $R^{2}$ & $F$ & $B$ & SE & beta & $t$ \\
\hline Acceptance & 0.130 & $5.31 * * *$ & & & & \\
\hline Months experience & & & 0.001 & 0.001 & 0.126 & 1.62 \\
\hline Weekly frequency & & & 0.057 & 0.023 & 0.187 & $2.46^{*}$ \\
\hline Average session duration & & & 0.008 & 0.003 & 0.188 & $2.67 * *$ \\
\hline Age & & & -0.006 & 0.006 & -0.077 & -1.04 \\
\hline \multirow[t]{2}{*}{ Education } & & & 0.126 & 0.071 & 0.127 & $1.77^{\dagger}$ \\
\hline & $R^{2}$ & $F$ & $B$ & SE & beta & $t$ \\
\hline Decentering/nonreact & 0.194 & $8.52 * * *$ & & & & \\
\hline Months experience & & & 0.001 & 0.001 & 0.103 & 1.37 \\
\hline Weekly frequency & & & 0.076 & 0.021 & 0.270 & $3.69 * * *$ \\
\hline Average session duration & & & 0.010 & 0.003 & 0.250 & $3.70 * * *$ \\
\hline Age & & & 0.001 & 0.005 & 0.019 & 0.27 \\
\hline \multirow[t]{2}{*}{ Education } & & & 0.090 & 0.063 & 0.099 & 1.43 \\
\hline & $R^{2}$ & $F$ & $B$ & SE & beta & $t$ \\
\hline Openness & 0.148 & $6.13 * * *$ & & & & \\
\hline Months experience & & & 0.001 & 0.001 & 0.103 & 1.33 \\
\hline Weekly frequency & & & 0.066 & 0.021 & 0.234 & $3.311 * *$ \\
\hline Average session duration & & & 0.008 & 0.003 & 0.212 & $3.05 * *$ \\
\hline Age & & & -0.005 & 0.005 & -0.069 & 0.95 \\
\hline \multirow[t]{2}{*}{ Education } & & & 0.100 & 0.066 & 0.108 & 1.52 \\
\hline & $R^{2}$ & $F$ & $B$ & $\mathrm{SE}$ & beta & $t$ \\
\hline Relativity of thoughts & 0.098 & $3.84 * *$ & & & & \\
\hline Months experience & & & 0.001 & 0.001 & 0.071 & 0.89 \\
\hline Weekly frequency & & & 0.043 & 0.020 & 0.168 & $2.718^{*}$ \\
\hline Average session duration & & & 0.007 & 0.003 & 0.187 & $2.61^{*}$ \\
\hline Age & & & -0.004 & 0.005 & -0.067 & -0.89 \\
\hline Education & & & 0.096 & 0.062 & 0.115 & 1.56 \\
\hline
\end{tabular}


Table 5 (continued)

\begin{tabular}{|c|c|c|c|c|c|c|}
\hline & $R^{2}$ & $F$ & $B$ & SE & beta & $t$ \\
\hline Insight & 0.118 & $4.74 * * *$ & & & & \\
\hline Months experience & & & 0.001 & 0.001 & 101 & 1.28 \\
\hline Weekly frequency & & & 0.056 & 0.020 & 0.208 & $2.72 * *$ \\
\hline Average session duration & & & 0.007 & 0.003 & 0.192 & $2.71 * *$ \\
\hline Age & & & -0.007 & 0.005 & -0.098 & -1.32 \\
\hline \multirow[t]{2}{*}{ Education } & & & 0.071 & 0.063 & 0.082 & 1.12 \\
\hline & $R^{2}$ & $F$ & $B$ & SE & beta & $t$ \\
\hline CHIME total & 0.202 & $8.97 * * *$ & & & & \\
\hline Months experience & & & 0.001 & 0.000 & 0.135 & $1.80^{\dagger}$ \\
\hline Weekly frequency & & & 0.057 & 0.015 & 0.279 & $3.83^{* * *}$ \\
\hline Average session duration & & & 0.007 & 0.002 & 0.234 & $3.47 * *$ \\
\hline Age & & & -0.002 & 0.004 & -0.029 & 0.42 \\
\hline Education & & & 0.072 & 0.046 & 0.108 & 1.57 \\
\hline
\end{tabular}

$n=183$; significance levels: ${ }^{\dagger} p<.10,{ }^{* *} p<.01, * * * p<.001$

significantly correlated with all CHIME subscales and average session duration was associated with all subscales except inner and outer awareness. When the relative weight of each of these meditation practice variables, together with age and education, were taken into account in the regression models, the predictive power of experience in months consistently dropped. Weekly frequency significantly predicted all but one (acting with awareness) subscale; average session duration was a significant predictor for all subscales but inner and outer awareness and acting with awareness, but meditation experience in months only predicted outer awareness scores. Finally, when different levels of practice and age were controlled for, no differences in levels of mindfulness could be established between three subgroups practicing either Vipassana meditation, Zen meditation, or body movementoriented techniques such as Yoga, Tai Chi, Qigong, and Kum Nye. Only in the CHIME subscales outer awareness and openness, differences at a trend level were found.
The present results emphasize the importance of an elaborate assessment of meditation practice. Most available studies operationalized meditation experience by the number of years of practice. According to the present results, however, the weekly frequency of practice shows even stronger associations with self-reported mindfulness. Moreover, in the correlation analyses, a practice variable that combined information on average session duration and frequency of practice yielded the strongest associations with mindfulness. This may be interpreted as showing the relatively high contribution of continued practice in the present, compared to the accumulated practice over years. This has implications for clinical intervention as it emphasizes the importance of a continued practice during and after the intervention. The results also suggest that, in spite of their differences in terms of technical execution and theoretical background, these techniques appear to equally impact the different aspects of mindfulness. This has

Table 6 ANCOVA analyses comparing mindfulness scores in meditators subgroups controlling for differences in meditation experience and age

\begin{tabular}{|c|c|c|c|c|c|c|}
\hline & \multicolumn{3}{|l|}{ Descriptives } & \multicolumn{3}{|c|}{ ANCOVA $F$ tests } \\
\hline & \multicolumn{3}{|l|}{ Mean (SD) } & \multirow{2}{*}{$\begin{array}{l}\text { Overall model } \\
F_{(5,89)} \\
\left(\mathrm{eta}^{2}\right)\end{array}$} & \multirow{2}{*}{$\begin{array}{l}\text { Factor technique } \\
F_{(2,92)} \\
\left(\mathrm{eta}^{2}\right)\end{array}$} & \multirow[t]{2}{*}{ Significant covariates } \\
\hline & Vipassana & Zen & Body-oriented & & & \\
\hline Inner awareness & $4.76(0.76)$ & $5.02(0.79)$ & $4.72(0.75)$ & $2.55^{*}(.127)$ & $0.19(.004)$ & Months experience* \\
\hline Outer awareness & $4.47(0.88)$ & $4.88(0.70)$ & $4.78(0.73)$ & $3.62 * *(.171)$ & $2.78^{\dagger}(.059)$ & Months experience* \\
\hline Acting with awareness & $4.26(0.85)$ & $4.44(0.72)$ & $4.16(0.82)$ & $2.16^{\dagger}(.109)$ & & \\
\hline Acceptance & $4.01(0.94)$ & $4.32(0.84)$ & $3.97(0.83)$ & $1.22(.065)$ & & \\
\hline Decentering/nonreact & $4.18(0.67)$ & $4.49(0.72)$ & $3.94(0.78)$ & $3.66 * *(.172)$ & $1.55(.034)$ & Average session duration* \\
\hline Openness & $4.32(0.94)$ & $4.68(0.74)$ & $3.91(0.99)$ & $3.23 *(.155)$ & $2.75^{\dagger}(.043)$ & Months experience* \\
\hline Relativity of thoughts & $4.93(0.75)$ & $4.95(0.76)$ & $4.73(0.66)$ & $1.07(.057)$ & & \\
\hline Insight & $4.37(0.81)$ & $4.55(0.82)$ & $4.29(0.73)$ & $1.33(.070)$ & & \\
\hline CHIME total score & $4.40(0.61)$ & $4.65(0.52)$ & $4.29(0.58)$ & $3.31 * *(.158)$ & $0.64(.014)$ & \\
\hline
\end{tabular}

$n=94$; Covariates: age, average session duration, month experience; significance levels: ${ }^{\dagger} p<.10,{ }^{*} p<.05,{ }^{* *} p<.01$ 
possible implications for interventions such as MBSR, which includes both sitting meditation and Hatha Yoga, in that participants might be even more encouraged to choose and concentrate on the techniques that better suit their personal preferences. This implication is nevertheless preliminary and should be corroborated in further research.

Bishop et al. (2004) proposed a two-component definition of mindfulness: differentiating self-regulation of attention and a particular orientation accompanying it; this orientation is characterized by curiosity, openness, and acceptance. The CHIME covers the former component, self-regulation of attention, with three variables: inner awareness, outer awareness, and acting with awareness. Of these, acting with awareness more strongly differentiated between meditators and nonmeditators. However, in a recent study, Soler et al. (2014) found stronger differences between non-meditators and meditators for the variable observe $(d=0.95, p<.001)$ than for acting with awareness $(d=0.24, p<.05)$. Within meditators, inner awareness was found to be more strongly associated with the amount of meditation practice, whereas acting with awareness showed the least associations. Concerning this latter aspect, previous studies provided mixed results: A number of studies could not establish a significant association between meditation practice and acting with awareness as measured by the KIMS, FFMQ, and MAAS, both in inexperienced samples and meditator samples (Baer et al. 2004, 2006, 2008; Falkenström 2010), whereas two studies pointed to significant associations in meditators (Brown and Ryan 2003; Falkenström 2010).

Five of the nine CHIME subscales, acceptance, decentering/nonreact, insight, relativity, and openness, cover the second component of mindfulness, its orientation. Some of these aspects are absent in the most widely used mindfulness questionnaires (i.e., MAAS, KIMS, and FFMQ). Within the meditator sample, decentering/nonreact emerged as an aspect that is importantly related to meditation practice, which may point to its central role in the development of mindfulness. This is supported by the results from the validation study of the TMS, which assesses decentering/nonreact and curiosity. Of both scales, decentering/nonreact was more strongly associated with meditation practice in both meditator subsamples in this study: MBSR and Shambhala meditators. The relevance of this aspect in the development of mindfulness is further substantiated by the model of the potential mechanisms of mindfulness proposed by Shapiro et al. (2006). In fact, within this model, "reperceiving," which involves decentering, deautomatization, detachment, and a fundamental shift in perspective, is described as a key transformative ingredient of mindfulness. It is also central in MBCT in which decentering is taught in order to reduce rumination and other automatic patterns (Baer et al. 2009). In the present data, relativity showed the least relationships with practice. Nevertheless, together with acceptance, relativity most strongly differentiated between non-meditators and meditators. It may thus be true that this aspect is of particular relevance at the beginning of meditation practice, even if the longterm impact of meditation training on it may be limited. Openness, insight, and acceptance were shown to be clearly related with meditation practice. The present results partly converge with those of the recent study by Soler et al. (2014), in which the strongest effects of meditation practice on mindfulness were found on the observing and nonreactivity subscales of the FFMQ and on the decentering subscale of the EQ, while weaker effects were established with the FFMQ subscales describing, acting with awareness, and non-judging. In contrast to results by Soler et al., in the present sample, not only months of experience and weekly frequency but also average session duration was significantly associated with most mindfulness subscales.

The current study has a number of limitations. First, its cross-sectional design does not allow making inferences on the causal relationships underlying the observed associations. Thus, these results are preliminary and need to be complemented by longitudinal designs. Mindfulness was solely assessed through self-report measures which, as discussed in the introduction, may limit the validity of the results. Significant age differences between the subgroups practicing either Zen, Vipassana, or body movementoriented techniques, as well as differences in their amount of practice, may have confounded the group comparisons. Moreover, the subgroup sizes were limited $(<40)$, which may have compromised power in ANCOVA analyses, and the body movement-oriented subgroup is less homogeneous in terms of included techniques than both the Zen and the Vipassana subgroups. Finally, there is room for improvement in the assessment of meditation practice. For example, variables related to current meditation practice (weekly frequency, average session duration) may be better comparable when a clear time frame is prescribed (e.g., "during the last two weeks").

Yet, the present study also has a number of strengths. It included large samples from the general population and MBSR groups. This allowed a detailed analysis of the relations between different variables of meditation practice, a broad coverage of aspects of mindfulness, and a comparison of different meditation techniques.

Further studies based on longitudinal designs providing a more complete, non-retrospective assessment of meditation practice over time are needed to elucidate causal connections and the mechanisms underlying these connections. Moreover, future studies may include the use of experimental and observational measures of mindfulness and thus not solely rely on self-report assessment. In fact, in response to the shortcomings of self-report assessment, alternative approaches to the assessment of mindfulness have been proposed. For example, the observational measure Assessment of Momentary Mindful 
Awareness (AMMA; Nyklíček and van Son 2013) is based on verbal expressions of momentary experience. The experimental measures Meditation Breath Attention Score (MBAS; Frewen et al. 2008, 2010) and Mindful-Breathing Exercise (MBE; Burg and Michalak 2011) are based on an individual's capacity to focus on breathing during a short period of time (10-20 min). Finally, effects of meditation practice on brain dynamics could be established within meditation states, but also at the trait level, which suggests the possibility to establish correlates of aspects of mindfulness such as changes in attentional allocation (Cahn and Polich 2006; Creswell et al. 2007). The joint use of such measures and self-report questionnaires may provide a richer and more faceted account of the development of mindfulness from the first- and the thirdperson perspective.

In summary, the present results provide further evidence for the relationships between self-report mindfulness and meditation practice. Results reveal that different patterns of associations between meditation practice and aspects of mindfulness can be found when meditation practice is measured in a more elaborate way. Consistent associations with mindfulness could be established with current meditation practice as well as with long-term practice, whereas results suggest that continued current practice is a better predictor of current mindfulness levels than the accumulated number of years of practice. Finally, quite heterogeneous meditation techniques were associated with comparable levels of mindfulness.

Acknowledgments The authors wish to thank the teachers of the Swiss MBSR Union for their valuable support of the study.

Conflict of Interest Authors declare no financial interests or benefit in the context of this study.

\section{References}

Baer, R. A., Smith, G. T., \& Allen, K. B. (2004). Assessment of mindfulness by self-report the Kentucky inventory of mindfulness skills. Assessment, 11, 191-206.

Baer, R. A., Smith, G. T., Hopkins, J., Krietemeyer, J., \& Toney, L. (2006). Using self-report assessment methods to explore facets of mindfulness. Assessment, 13, 27-45.

Baer, R. A., Smith, G. T., Lykins, E., Button, D., Krietemeyer, J., Sauer, S., et al. (2007). Construct validity of the Five Facet Mindfulness Questionnaire in meditating and nonmeditating samples. Assessment, 15, 329-342.

Baer, R. A., Smith, G. T., Lykins, E., Button, D., Krietemeyer, J., Sauer, S., Walsh, E., Duggan, D., \& Williams, J. M. G. (2008). Construct validity of the five facet mindfulness questionnaire in meditating and nonmeditating samples. Assessment, 15, 329-342.

Baer, R. A., Walsh, E., \& Lykins, E. L. (2009). Assessment of mindfulness. In F. Didonna (Ed.), Clinical handbook of mindfulness (pp. 153-168). New York: Springer.

Belzer, F., Schmidt, S., Lucius-Hoene, G., Schneider, J. F., Orellana-Rios, C. L., \& Sauer, S. (2013). Challenging the construct validity of mindfulness assessment - a cognitive interview study of the Freiburg mindfulness inventory. Mindfulness, 4, 33-44.

Bergomi, C., Tschacher, W., \& Kupper, Z. (2013). Measuring mindfulness: first steps towards the development of a comprehensive mindfulness scale. Mindfulness, 4, 18-32.

Bergomi, C., Tschacher, W., \& Kupper, Z. (2014). Konstruktion und erste Validierung eines Fragebogens zur umfassenden Erfassung von Achtsamkeit: Das Comprehensive Inventory of Mindfulness Experiences. Diagnostica, 60, 111-125.

Bishop, S. R., Lau, M., Shapiro, S., Carlson, L., Anderson, N. D., Carmody, J., Segal, Z. V., Abbey, S., Speca, M., Velting, D., \& Devins, G. (2004). Mindfulness: a proposed operational definition. Clinical Psychology: Science and Practice, 11, 230-241.

Brown, K. W., \& Ryan, R. M. (2003). The benefits of being present: mindfulness and its role in psychological well-being. Journal of Personality and Social Psychology, 84, 822.

Brown, K. W., \& Ryan, R. M. (2004). Perils and promises in defining and measuring mindfulness: observations from experience. Clinical Psychology: Science and Practice, 11, 242-248.

Burg, J. M., \& Michalak, J. (2011). The healthy quality of mindful breathing: associations with rumination and depression. Cognitive Therapy and Research, 35, 179-185.

Cahn, B. R., \& Polich, J. (2006). Meditation states and traits: EEG, ERP, and neuroimaging studies. Psychological Bulletin, 132, 180.

Caldwell, K., Harrison, M., Adams, M., Quin, R. H., \& Greeson, J. (2010). Developing mindfulness in college students through movement-based courses: effects on self-regulatory self-efficacy, mood, stress, and sleep quality. Journal of American College Health, 58, 433-442.

Carmody, J., \& Baer, R. A. (2008). Relationships between mindfulness practice and levels of mindfulness, medical and psychological symptoms and well-being in a mindfulness-based stress reduction program. Journal of Behavioral Medicine, 31, 23-33.

Chadwick, P., Hember, M., Symes, J., Peters, E., Kuipers, E., \& Dagnan, D. (2008). Responding mindfully to unpleasant thoughts and images: reliability and validity of the Southampton Mindfulness Questionnaire (SMQ). British Journal of Clinical Psychology, 47, 451-455.

Chiesa, A. (2013). The difficulty of defining mindfulness: current thought and critical issues. Mindfulness, 4, 255-268.

Christopher, M. S., Christopher, V., \& Charoensuk, S. (2009). Assessing "Western" mindfulness among Thai Theravāda Buddhist Monks. Mental Health, Religion and Culture, 12, 303-314.

Christopher, M. S., Woodrich, L. E., \& Tiernan, K. A. (2014). Using cognitive interviews to assess the cultural validity of state and trait measures of mindfulness among Zen Buddhists. Mindfulness, 5, 145-160.

Creswell, J. D., Way, B. M., Eisenberger, N. I., \& Lieberman, M. D. (2007). Neural correlates of dispositional mindfulness during affect labeling. Psychosomatic Medicine, 69, 560-565.

Falkenström, F. (2010). Studying mindfulness in experienced meditators: a quasi-experimental approach. Personality and Individual Differences, 48, 305-310.

Fresco, D. M., Moore, M. T., van Dulmen, M. H., Segal, Z. V., Ma, S. H., Teasdale, J. D., \& Williams, J. M. G. (2007). Initial psychometric properties of the experiences questionnaire: validation of a selfreport measure of decentering. Behavior Therapy, 38, 234-246.

Frewen, P. A., Evans, E. M., Maraj, N., Dozois, D. J. A., \& Partridge, K. (2008). Letting go: mindfulness and negative automatic thinking. Cognitive Therapy and Research, 32, 758-774.

Frewen, P. A., Dozois, D. J. A., Neufeld, R. W. J., Lane, R. D., Densmore, M., Stevens, T. K., et al. (2010). Individual differencesin trait mindfulness predict dorsomedial prefrontal and amygdalaresponse during emotional imagery: an fMRI study. Personality and Individual Differences, 49, 479-484. 
Grossman, P. (2008). On measuring mindfulness in psychosomatic and psychological research. Journal of Psychosomatic Research, 64, 405-408.

Grossman, P. (2010). Mindfulness for psychologists: paying kind attention to the perceptible. Mindfulness, 1, 87-97.

Grossman, P. (2011). Defining mindfulness by how poorly I think I pay attention during everyday awareness and other intractable problems for psychology's (re)invention of mindfulness: Comment on Brown et al. (2011). Psychological Assessment, 23, 1034-1040.

Kabat-Zinn, J. (1994). Wherever you go, there you are: mindfulness meditation in everyday life. New York: Hyperion.

Kabat-Zinn, J. (2003). Mindfulness-based interventions in context: past, present, and future. Clinical Psychology: Science and Practice, 10, 144-156.

Lau, M., Bishop, S. R., Segal, Z. V., Buis, T., Anderson, N. D., Carlson, L., Shapiro, S., Carmody, J., Abbey, S., \& Devins, G. (2006). The Toronto mindfulness scale: development and validation. Journal of Clinical Psychology, 62, 1445-1467.

Nyklíček, I. \& van Son, J. (2013). An Observational Measure of Mindful Awareness: Validation of the Assessment of Momentary Mindful Awareness (AMMA). Paper presented at the First International Conference on Mindfulness, Rome, Italy.

Rosch, E. (2007). More than mindfulness: when you have a tiger by the tail, let it eat you. Psychological Inquiry, 18, 258-264.

Schure, M. B., Christopher, J., \& Christopher, S. (2008). Mind-body medicine and the art of self-care: teaching mindfulness to counseling students through yoga, meditation, and qigong. Journal of Counseling and Development, 86, 47-56.

Shapiro, S. L., Carlson, L. E., Astin, J. A., \& Freedman, B. (2006). Mechanisms of mindfulness. Journal of Clinical Psychology, 62, 373-386.

Shelov, D. V., Suchday, S., \& Friedberg, J. P. (2009). A pilot study measuring the impact of yoga on the trait of mindfulness. Behavioural and Cognitive Psychotherapy, 37, 595-598.

Soler, J., Cebolla, A., Feliu-Soler, A., Demarzo, M. M., Pascual, J. C., Baños, R., \& García-Campayo, J. (2014). Relationship between meditative practice and self-reported mindfulness: the MINDSENS composite index. PloS One, 9, e86622.

Sugiura, Y., Sato, A., Ito, Y., \& Murakami, H. (2012). Development and validation of the Japanese version of the Five Facet Mindfulness Questionnaire. Mindfulness, 3, 85-94.

Thompson, B. L., \& Waltz, J. (2010). Mindfulness and experiential avoidance as predictors of posttraumatic stress disorder avoidance symptom severity. Journal of Anxiety Disorders, 24, 409-415.

Van Dam, N., Earleywine, M., \& Danoff-Burg, S. (2009). Differential item function across meditators and non-meditators on the Five Facet Mindfulness Questionnaire. Personality and Individual Differences, 47, 516-521.

Walach, H., Buchheld, N., Buttenmüller, V., Kleinknecht, N., \& Schmidt, S. (2006). Measuring mindfulness-The Freiburg mindfulness inventory (FMI). Personality and Individual Differences, 40, 15431555 . 